\title{
Queueing System with Heterogeneous Customers as a Model of a Call Center with a Call-Back for Lost Customers
}

\author{
Sergey Dudin, ${ }^{1}$ Chesoong Kim, ${ }^{2}$ Olga Dudina, ${ }^{1}$ and Janghyun Baek ${ }^{3}$ \\ ${ }^{1}$ Belarusian State University, 4 Nezavisimosti Avenue, 220030 Minsk, Belarus \\ ${ }^{2}$ Sangji University, Wonju, Kangwon 220-702, Republic of Korea \\ ${ }^{3}$ Chonbuk National University, Jeonju 561-756, Republic of Korea \\ Correspondence should be addressed to Chesoong Kim; dowoo@sangji.ac.kr
}

Received 2 July 2013; Accepted 29 September 2013

Academic Editor: Gianluca Ranzi

Copyright (C) 2013 Sergey Dudin et al. This is an open access article distributed under the Creative Commons Attribution License, which permits unrestricted use, distribution, and reproduction in any medium, provided the original work is properly cited.

A multiserver queueing system with infinite and finite buffers, two types of customers, and two types of servers as a model of a call center with a call-back for lost customers is investigated. Type 1 customers arrive to the system according to a Markovian arrival process. All rejected type 1 customers become type 2 customers. Type $r, r=1,2$, servers serve type $r$ customers if there are any in the system and serve type $r^{\prime}, r^{\prime}=1,2, r^{\prime} \neq r$, customers if there are no type $r$ customers in the system. The service times of different types of customers have an exponential distribution with different parameters. The steady-state distribution of the system is analyzed. Some key performance measures are calculated. The Laplace-Stieltjes transform of the sojourn time distribution of type 2 customers is derived. The problem of optimal choice of the number of each type servers is solved numerically.

\section{Introduction}

A call center is a specialized unit of companies that handles voice requests from clients. As call centers provide a communication channel between companies and their customers, the effective call center performance is extremely important for company's reputation. For modeling and optimizing call centers, the queueing theory is used. For background information and an overview of the present state of the art in the study of call centers, the reader is referred to the survey [1] and the papers [2,3], as well as references therein.

It is not a secret that there is a high competition between companies, and a customer, who does not receive service in a call center of one company, can simply move to competitors' company. Loss of customers or potential customers may cause damage or loss of profit. This is especially important for such organizations as banks, Internet shops, telemarkets, and other organizations that deal with selling and providing the paid services. For these organizations, loss of calls causes loss of clients and increases the risk of clients dealing with competitors. For example, if a potential client, who wants to get the information about deposits, does not succeed in connecting with an operator of a call center of one bank, this client can contact another bank and open a deposit there. Thus, the issue of effective service of calls with minimum losses of customers is very important. At the same time, to achieve the call center performance without losses is very difficult because

(i) the arrival flow of customers can be correlated (the time intervals in which customers arrive in the system often alternate with intervals in which few customers arrive in the system);

(ii) it is too expensive to have a large number of operators (most of the costs of a call center involve wages).

So, it seems to be reasonable to call back a customer, who did not wait for the answer of an operator of a call center, 
and offer him (her) the services. We assume that if customer leaves the system without service, his (her) phone number is added to some infinite size virtual queue, and an operator calls back him (her) later. Let us refer to a customer who leaves the system without service as a virtual customer and a customer who waits on hold as a real customer. One way is to provide a call-back to virtual customers when there is an idle operator and there are no customers waiting on hold. But in this case, the waiting time of a call-back can be very long and the customer will no longer need the services provided by the call center. In our paper, we consider more reasonable and general way to provide a call-back. We assume that all call center operators are divided into two groups. For the first group of operators (type 1), processing of real customers has a priority, while the operators from the second group (type 2) firstly provide service to virtual customers, if there are any in the system. This approach allows us to solve the issue of determination of the number of different types of operators under constraints on the waiting time of an arbitrary virtual customer but complicates the investigation of the system.

Models of call centers with a call-back option were considered in [4-6]. In all these models, a call-back was considered as an option which is proposed to a customer. We consider the model of a call center in which a call-back is not proposed to the customers but operators who call back all customers leaving the system without service. In $[4,5]$, an asymptotic analysis of the model of a call center with a callback option and identical servers in the case of heavy load for a large number of operators is presented. In our paper, we consider more general customers' arrival process and the possibility of customers abandonment. Also, we present an exact analytical analysis of the model without the restriction that the number of operators is sufficiently large. In [6], all operators are assumed to be identical and call-backs to virtual customers are provided only when there is an idle operator and there are no real customers in the system. In our paper, we considered a more general and complicated model where all operators are divided into groups.

The paper is organized as follows. In Section 2, the mathematical model is described. The ergodicity condition and the stationary distribution of the system states are analyzed in Section 3. The expressions for the main performance measures of the system are given in Section 4. The LaplaceStieltjes transform of the sojourn time distribution of an arbitrary virtual customer is presented in Section 5. Section 6 contains numerical illustrations and their short discussion. Section 7 concludes the paper.

\section{Mathematical Model}

We consider an $N$-server queuing system with two types of customers, a buffer of capacity $K$ for real customers, and an infinite buffer for virtual customers to model a call center with a call-back option. The structure of the system under study is presented in Figure 1.

Real (type 1) customers arrive at the system according to a Markovian arrival process (MAP). The MAP is defined

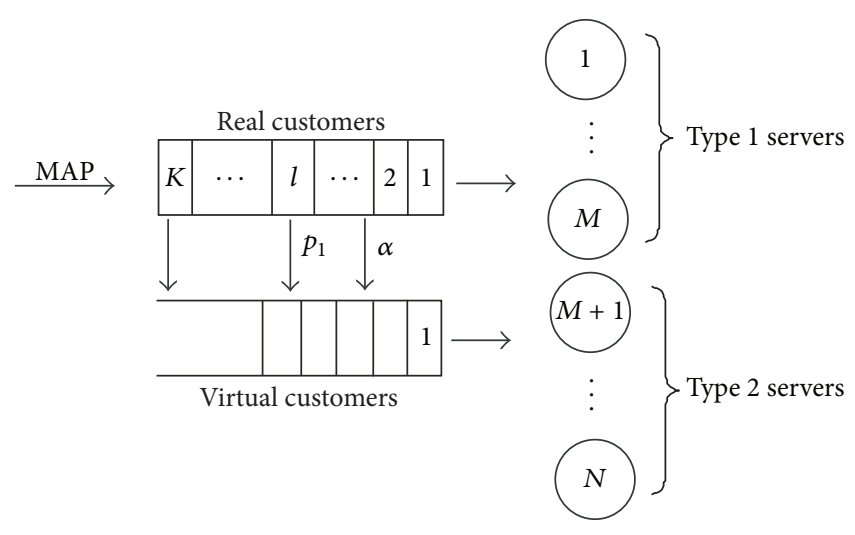

FIGURE 1: Structure of the system.

by the underlying process $v_{t}, t \geq 0$, which is an irreducible continuous-time Markov chain with the state space $\{0,1, \ldots, W\}$. Arrivals occur only at the epochs of transitions in the underlying process $v_{t}, t \geq 0$. The intensities of transitions of the process $v_{t}, t \geq 0$, that are accompanied (not accompanied) by the arrival of a customer are defined by the square matrix $D_{1}\left(D_{0}\right)$ of size $\bar{W}=W+1$.

The matrix $D(1)=D_{0}+D_{1}$ is an infinitesimal generator of the process $v_{t}, t \geq 0$. The stationary distribution vector $\boldsymbol{\theta}$ of this process satisfies the system of equations $\boldsymbol{\theta} D(1)=\mathbf{0}$, $\boldsymbol{\theta} \mathbf{e}=1$. Here and throughout this paper, $\mathbf{0}$ is a zero row vector, and $\mathbf{e}$ denotes a unit column vector.

The average intensity $\lambda$ (fundamental rate) of the MAP is defined by $\lambda=\theta D_{1}$ e. The coefficient of variation $c_{\text {var }}$ of intervals between customer arrivals is calculated as $c_{\mathrm{var}}^{2}=2 \lambda \boldsymbol{\theta}\left(-D_{0}\right)^{-1} \mathbf{e}-1$. The coefficient of correlation $c_{\mathrm{cor}}$ of intervals between successive arrivals is given as $c_{\text {cor }}=$ $\left(\lambda \boldsymbol{\theta}\left(-D_{0}\right)^{-1}\left(D(1)-D_{0}\right)\left(-D_{0}\right)^{-1} \mathbf{e}-1\right) / c_{\text {var }}^{2}$

If there is an available server during an arbitrary real customer arrival epoch, this customer is admitted into the system and occupies a free type 1 server if there is, or a type 2 server, otherwise. If all servers are busy during a real customer arrival epoch, this customer becomes aware of the current queue length ("visible" queue) and, based on the information provided, decides whether to wait in line or leave the system (balk). Thus, if all servers are busy and there are $k, k=$ $\overline{0, K-1}$, real customers in the finite buffer during an arbitrary real customer arrival epoch, the arriving customer leaves the system with probability $p_{k}$, and with the complementary probability the customer chooses not to wait for service. If the buffer for type l customers is full during an arbitrary customer arrival epoch, this customer always leaves the system; that is, $p_{K}=1$.

Real customers are impatient; that is, a real customer leaves the buffer after an exponentially distributed time described by the parameter $\alpha, 0<\alpha<\infty$, due to a lack of service.

We assume that, if a real customer leaves the system without service, this customer changes its type (becomes a virtual customer) and joins a virtual waiting place of infinite size (infinite buffer). Virtual customers are always patient. We 
assume that both types of customers are served according to the First In-First Out rule. The difference between two types of customers is that a real customer physically presents in the system and holds a line, while a virtual customer is not presented in the system.

All servers (operators) are divided into two types:

(1) type 1 operators who serve the real customers;

(2) type 2 operators who call back the customers who left the system (virtual customers).

The number of type 1 servers is $M, M=\overline{0, N}$, and the number of type 2 servers is $N-M$.

If a type 1 operator becomes idle and there are no real customers in the queue but there are virtual customers who wait for a call-back, this operator starts to serve a virtual customer to avoid the idle time. Similarly, if a type 2 operator becomes idle and there are no virtual customers in the queue but there are real customers waiting for service, then this operator starts to serve a real customer.

The service times of real and virtual customers have an exponential distribution with the parameters $\mu_{1}$ and $\mu_{2}$, respectively.

\section{The Process of System States}

Let $i_{t}$ be the number of customers in the system, $k_{t}$ the number of real customers in the finite buffer, $m_{t}$ the number of busy type 1 servers, $n_{t}$ the number of busy type 1 servers that serve the real customers, $l_{t}$ the number of busy type 2 servers that serve the real customers, and $v_{t}$ the state of the underlying process of the MAP during the epoch $t, t \geq 0$.

The behavior of the system under consideration can be described in terms of the regular irreducible continuous-time
Markov chain $\xi_{t}=\left\{i_{t}, k_{t}, m_{t}, n_{t}, l_{t}, v_{t}\right\}, t \geq 0$, with the state space

$$
\begin{aligned}
& (\{i, m, n, l, v\}, i<N, \\
& m=\overline{\max \{0, i-(N-M)\}, \min \{i, M\}}, \\
& n=\overline{0, m}, l=\overline{0, \min \{i-m, N-M\}}, v=\overline{0, W}) \\
& \quad \bigcup(\{i, k, n, l, v\}, i \geq N, k=\overline{0, \min \{i-N, K\}} \\
& \quad n=\overline{0, M}, l=\overline{0, N-M}, v=\overline{0, W}) .
\end{aligned}
$$

Let us introduce the operator $\mathscr{T}\left(A_{k, k-1}, k=\overline{k_{1}, K_{1}}\right.$; $\left.A_{k, k}, k=\overline{k_{2}, K_{2}} ; A_{k, k+1}, k=\overline{k_{3}, K_{3}}\right)$ which define a block tridiagonal matrix with subdiagonal blocks $A_{k, k-1}$, diagonal blocks $A_{k, k}$, and updiagonal blocks $A_{k, k+1}$. By default, we assume that this block matrix is square or the number of its block rows is equal to the number of block columns \pm 1 .

Let us enumerate states of Markov chain $\xi_{t}$ in the lexicographic order of the components $k, m, n, l$, and $\nu$. Let $Q$ be a generator of the Markov chain $\xi_{t}, t \geq 0$, consisting of the blocks $Q_{i, j}$, which define the transition rates of this chain from the states having value $i$ of the first component to the states having value $j$ of this component of the chain. The diagonal entries of the matrices $Q_{i, i}$ are negative, and the modulus of diagonal entries defines the total intensity of leaving the corresponding state of the Markov chain $\xi_{t}, t \geq 0$.

Lemma 1. The infinitesimal generator $Q$ of the Markov chain $\xi_{t}, t \geq 0$, has a block tridiagonal structure:

$$
Q=\left(\begin{array}{ccccccccccc}
Q_{0,0} & Q_{0,1} & O & O & \cdots & O & O & O & O & O & \cdots \\
Q_{1,0} & Q_{1,1} & Q_{1,2} & O & \cdots & O & O & O & O & O & \cdots \\
O & Q_{2,1} & Q_{2,2} & Q_{2,3} & \cdots & O & O & O & O & O & \cdots \\
\vdots & \vdots & \ddots & \ddots & \ddots & \vdots & \vdots & \vdots & \vdots & \vdots & \cdots \\
O & O & O & O & \cdots & Q_{N+K-1, N+K-2} & Q_{N+K-1, N+K-1} & Q_{N+K-1, N+K} & O & O & \cdots \\
O & O & O & O & \cdots & O & Q_{N+K, N+K-1} & Q_{1} & Q_{2} & O & \cdots \\
O & O & O & O & \cdots & O & O & Q_{0} & Q_{1} & Q_{2} & \cdots \\
\vdots & \cdots & \vdots & \vdots & \vdots & \vdots & \vdots & \vdots & \ddots & \ddots & \ddots
\end{array}\right)
$$

The nonzero blocks $Q_{i, j}, i, j \geq 0$, have the following form:

$$
\begin{array}{r}
Q_{i, i}=\operatorname{diag}\{\operatorname{diag}\{\operatorname{diag}\{A(i, m, n, l), \\
\quad l=\overline{0, \min \{N-M, i-m\}}\}, \\
\left.n=\overline{0, m}\}, m=\overline{i^{*}, \min \{i, M\}}\right\}, \\
\quad i=\overline{0, N-1},
\end{array}
$$

$$
\begin{gathered}
Q_{i, i}=\mathscr{T}\left(B_{k, k-1}, k=\overline{1, i-N} ; B_{k, k}, k=\overline{0, i-N} ;\right. \\
\left.O_{k}, k=\overline{0, i-N-1}\right), \quad i=\overline{N, N+K-1}, \\
Q_{1}=\mathscr{T}\left(B_{k, k-1}, k=\overline{1, K} ; B_{k, k}, k=\overline{0, K} ;\right. \\
\left.O_{k}, k=\overline{0, K-1}\right), \quad i \geq N+K,
\end{gathered}
$$




$$
\begin{aligned}
& Q_{i, i+1}=\left\{\begin{aligned}
& \mathscr{T}\left(O_{m}, m=\overline{1, i} ; O_{m, m}^{(i)}, m=\overline{0, i}\right. \\
&\left.C_{m, m+1}^{(i)}, m=\overline{0, i}\right), i<N-M ; \\
& \operatorname{diag}\left\{C_{m, m+1}^{(i)}, m=\overline{i^{*}, i}\right\}, i \geq N-M, \\
& i=\overline{0, M-1},
\end{aligned}\right. \\
& \left\{\begin{array}{c}
\mathscr{T}\left(O_{m}, m=\overline{1, M} ; C_{m, m}^{(i)}, m=\overline{0, M}\right. \\
\left.C_{m, m+1}^{(i)}, m=\overline{0, M-1}\right)
\end{array}\right. \\
& Q_{i,+1}=\left\{\begin{array}{r}
i<N-M \\
\mathscr{T}\left(C_{m, m}^{(i)}, m=\overline{i^{*}+1, M}\right.
\end{array}\right. \\
& C_{m, m+1}^{(i)}, m=\overline{i^{*}, M-1} ; \\
& \left.O_{m}, m=\overline{i^{*}, M-2}\right), \\
& i \geq N-M \\
& i=\overline{M, N-1} \\
& Q_{i, i+1}=\mathscr{T}\left(O_{k}, k=\overline{1, i-N} ; H_{k, k}, k=\overline{0, i-N}\right. \\
& \left.H_{k, k+1}, k=\overline{0, i-N}\right), \quad i=\overline{N, N+K-1}, \\
& Q_{2}=\mathscr{T}\left(O_{k}, k=\overline{1, K} ; H_{k, k}, k=\overline{0, K}\right.
\end{aligned}
$$$$
\left.H_{k, k+1}, k=\overline{0, K-1}\right), \quad i \geq N+K,
$$$$
Q_{i, i-1}=\left\{\begin{array}{rr}
\mathscr{T}\left(E_{m, m-1}^{(i)}, m=\overline{1, i} ; E_{m, m}^{(i)}, m=\overline{0, i-1}\right. \\
\left.O_{m}, m=\overline{0, i-2}\right), \quad i \leq N-M ; \\
\mathscr{T}\left(O_{m}, m=\overline{i^{*}+1, i} ; E_{m, m-1}^{(i)}, m=\overline{i^{*}, i} ;\right. \\
\left.E_{m, m}^{(i)}, m=\overline{i^{*}, i-1}\right), \quad i>N-M, \\
i=\overline{1, M},
\end{array}\right.
$$

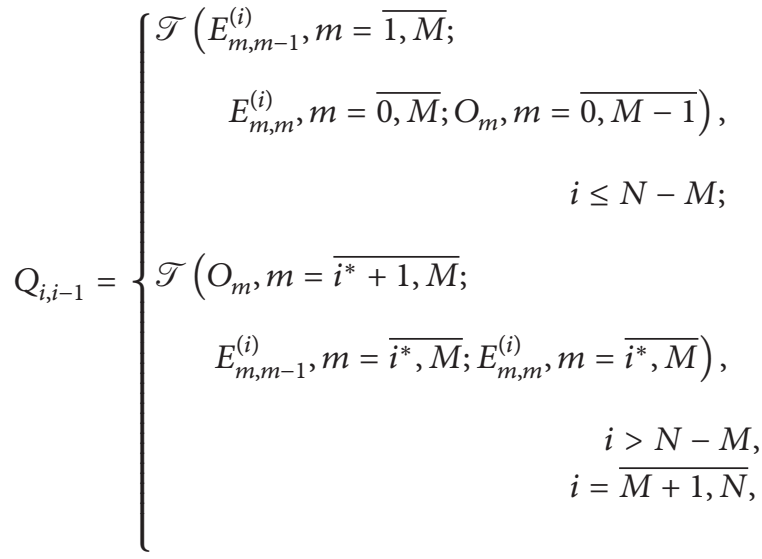

$$
\begin{aligned}
& Q_{i, i-1}=\mathscr{T}\left(Z_{k, k-1}^{(i)}, k=\overline{1, i-N} ; Z_{k, k}, k=\overline{0, i-N-1} ;\right. \\
& \left.O_{k}, k=\overline{0, i-N-2}\right), \quad i=\overline{N+1, N+K}, \\
& Q_{0}=\mathscr{T}\left(V_{k, k-1}, k=\overline{1, K} ; V_{k, k}, k=\overline{0, K}\right. \\
& \left.\mathrm{O}_{k}, k=\overline{0, K-1}\right), \quad i>N+K \text {. }
\end{aligned}
$$

Here, $I$ is the identity matrix, $O$ is a zero matrix of appropriate dimension, and $\mathrm{O}_{l}, l=\overline{k, K}$, is the sequence which consists of $K-k+1$ zero matrices of appropriate dimension:

$$
i^{*}=\max \{0, i-(N-M)\} .
$$

$\otimes$ indicates the Kronecker product of matrices:

$A(i, m, n, l)$

$$
=\left\{\begin{array}{cc}
-\left[(n+l) \mu_{1}\right. & \\
\left.+(i-n-l) \mu_{2}\right] I_{\bar{W}}+D_{0}, & i-m \leq N-M, \\
D_{0}, & i-m>N-M
\end{array}\right.
$$

$$
\begin{aligned}
& B_{k, k}=-B \otimes I_{\bar{W}}+I_{(M+1)(N-M+1)} \otimes\left(-k \alpha I_{\bar{W}}+D_{0}\right) \\
& \text { where } B=\operatorname{diag}\left\{\operatorname { d i a g } \left\{(n+l) \mu_{1}+(N-n-l) \mu_{2}, l=\right.\right. \\
& \overline{0, N-M}\}, n=\overline{0, M}\} ; \\
& \qquad B_{k, k-1}=k \alpha I_{(M+1)(N-M+1) \bar{W}} ; \\
& \frac{C_{m, m+1}^{(i)}=\mathscr{T}\left(O_{n}, n=\overline{1, m} ; O_{n}, n=\overline{0, m} ; C_{n, n+1}^{(i, m)}, n=\right.}{0, m) \text { where } C_{n, n+1}^{(i, m)}=I_{\min \{i-m, N-M\}+1} \otimes D_{1} ;}
\end{aligned}
$$

$$
C_{m, m}^{(i)}= \begin{cases}O_{(i+1) \bar{W} \times(i+2) \bar{W}}, & m=0, i<N-M \\ O_{(N-M+1) \bar{W} \times(N-M+1) \bar{W}}, & m=0, i \geq N-M \\ O_{(m+1)(\min \{i-m, N-M\}+1) \bar{W} \times(m+1)(\min \{i-m+1, N-M\}+1) \bar{W}}, & m=\overline{1, M-1} \\ I_{M+1} \otimes I_{i}^{+} \otimes D_{1}, & m=M,\end{cases}
$$


where $I_{i}^{+}$is a matrix of size $(i-M+1) \times(i-M+2)$ with nonzero entries $\left(I_{i}^{+}\right)_{l, l+1}, l=\overline{0, i-M}$, which are equal to 1 . Consider

$$
\begin{aligned}
& O_{m, m}^{(i)}= \begin{cases}O_{(i+1) \bar{W} \times(i+2) \bar{W}}, & m=0, \\
O_{(m+1)(\min \{i-m, N-M\}+1) \bar{W} \times(m+1)(\min \{i-m+1, N-M\}+1) \bar{W},} & m=\overline{1, i} ;\end{cases} \\
& H_{k, k}=I_{(M+1)(N-M+1)} \otimes p_{k} D_{1} ; H_{k, k+1}=I_{(M+1)(N-M+1)} \otimes\left(1-p_{k}\right) D_{1} ; \\
& E_{m, m}^{(i)}=I_{m+1} \otimes \mathscr{T}\left(\mu_{1} l, l=\overline{1, i-m} ; \mu_{2}(i-m-l), l=\overline{0, i-m-1} ; O_{l}, l=\overline{0, i-m-2}\right) \otimes I_{\bar{W}} ; \\
& E_{m, m-1}^{(i)}=\mathscr{T}\left(\mu_{1} l, l=\overline{1, m} ; \mu_{2}(m-l), l=\overline{0, m-1} ; O_{l}, l=\overline{0, m-2}\right) \otimes I_{(i-m+1) \bar{W}} ; \\
& Z_{k, k}= \begin{cases}\mathscr{T}\left(\mu_{1} l I_{\bar{W}(N-M+1)}, l=\overline{1, M} ; \Psi+\mu_{2}(M-l) I_{(N-M+1) \bar{W}}, l=\overline{0, M} ;\right. & \\
\left.\quad O_{l}, l=\overline{0, M-1}\right), & k=0 ; \\
I_{M+1} \otimes \Psi, & k=\overline{1, K-1,}\end{cases} \\
& \Psi=\mathscr{T}\left(\mu_{1} l, l=\overline{1, N-M} ; \mu_{2}(N-M-l), l=\overline{0, N-M} ; O_{l}, l=\overline{0, N-M-1}\right) \otimes I_{\bar{W}} ; \\
& Z_{k, k-1}^{(i)}=\left\{\begin{aligned}
\Phi, & k=\overline{1, i-N} \Phi+I_{M+1} \otimes \mathscr{T}\left(O_{l}, l=\overline{1, N-M} ; \mu_{1} l, l=\overline{0, N-M} ;\right. \\
\left.\mu_{2}(N-M-l), l=\overline{0, N-M-1}\right) \otimes I_{\bar{W}}, & k=i-N,
\end{aligned}\right. \\
& \Phi=\mathscr{T}\left(O_{n}, n=\overline{1, M} ; \mu_{1} n, n=\overline{0, M} ; \mu_{2}(M-n), n=\overline{0, M-1}\right) \otimes I_{(N-M+1) \bar{W}} ; \\
& V_{k, k}= \begin{cases}\mathscr{T}\left(\mu_{1} n I_{N-M+1}, n=\overline{1, M} ; \bar{V}_{n, n}, n=\overline{0, M} ; O_{n}, n=\overline{0, M-1}\right) \otimes I_{\bar{W}}, & k=0, \\
I_{M+1} \otimes \Psi, & k=\overline{1, K,}\end{cases}
\end{aligned}
$$

where $\bar{V}_{n, n}=\mathscr{T}\left(\mu_{1} l, l=\overline{1, N-M} ; \mu_{2}(N-n-l), l=\right.$ $\left.\overline{0, N-M} ; O_{l}, l=\overline{0, N-M}\right)$. Consider

$$
\begin{aligned}
V_{k, k-1}=\mathscr{T}\left(O_{n}, n=\overline{1, M} ; \mu_{1} n, n=\overline{0, M}\right. & \\
& \left.\mu_{2}(M-n), n=\overline{0, M-1}\right) \otimes I_{(N-M+1) \bar{W}} .
\end{aligned}
$$

The proof of the lemma is implemented by the analysis of all transitions of the Markov chain $\xi_{t}, t \geq 0$, during the interval of infinitesimal length and rewriting the intensities of these transitions in block matrix form.

Corollary 2. The Markov chain $\xi_{t}, t \geq 0$, belongs to the class of continuous time quasi-birth-and-death processes; see, for example, [7].

Theorem 3. The Markov chain $\xi_{t}, t \geq 0$, is ergodic if the following inequality holds true:

$$
\mathbf{y} \mathscr{A}\left(B \otimes I_{\bar{W}}\right) \mathbf{e}>\mathbf{y} \mathscr{A}\left(I_{M+1} \otimes D_{1}\right) \mathbf{e},
$$

where the vector $\mathbf{y}$ is the unique solution to the system

$$
\mathbf{y}\left(\bar{Q}_{0,0}+T_{0} \bar{Q}_{1,0}\right)=\mathbf{0}, \quad \mathbf{y}(I+\mathscr{A}) \mathbf{e}=1 .
$$

Here,

$$
\mathscr{A}=\sum_{k=0}^{K} \prod_{l=0}^{k} T_{l}
$$

the matrices $T_{k}, k=\overline{0, K-1}$, are calculated using the backward recursion

$$
\begin{gathered}
T_{K-1}=-\bar{Q}_{K-1, K}\left(\bar{Q}_{K, K}\right)^{-1}, \\
T_{k}=-\bar{Q}_{k, k+1}\left(\bar{Q}_{k+1, k+1}+T_{k+1} \bar{Q}_{k+2, k+1}\right)^{-1}, \\
k=K-2, K-3, \ldots, 0 ;
\end{gathered}
$$

the matrices $\bar{Q}_{k, k}, \bar{Q}_{k, k+1}$, and $\bar{Q}_{k, k-1}$ are defined as follows:

$$
\begin{gathered}
\bar{Q}_{0,0}=\mathscr{T}\left(\mu_{1} n, n=\overline{1, M} ;-\mu_{1} n,\right. \\
\left.n=\overline{0, M} ; O_{n}, n=\overline{0, M-1}\right) \\
\otimes I_{\bar{W}}+I_{M+1} \otimes\left(D_{0}+p_{0} D_{1}\right), \\
\bar{Q}_{k, k}=\operatorname{diag}\left\{-\mu_{1} n-\mu_{2}(M-n), n=\overline{0, M}\right\} \\
\otimes I_{\bar{W}}-k \alpha I_{(M+1) \bar{W}}+I_{M+1} \otimes\left(D_{0}+p_{k} D_{1}\right), \\
\quad k=\overline{1, K}, \\
\bar{Q}_{k, k+1}=\left(1-p_{k}\right)\left(I_{M+1} \otimes D_{1}\right), \quad k=\overline{0, K-1,}
\end{gathered}
$$




$$
\begin{aligned}
& \bar{Q}_{k, k-1}= k \alpha I_{(M+1) \bar{W}} \\
&+\mathscr{T}\left(O_{n}, n=\overline{1, M} ; \mu_{1} n, n=\overline{0, M} ;\right. \\
&\left.(M-n) \mu_{2}, n=\overline{0, M-1}\right) \otimes I_{\bar{W}} \\
& k=\overline{1, K} .
\end{aligned}
$$

Proof. It follows from [7] that the necessary and sufficient ergodicity condition of the quasi-birth-and-death process is the fulfillment of the inequality

$$
\mathbf{x} Q_{0} \mathbf{e}>\mathbf{x} Q_{2} \mathbf{e},
$$

where the vector $\mathbf{x}$ is the unique solution to the following system of linear algebraic equations:

$$
\mathbf{x}\left(Q_{0}+Q_{1}+Q_{2}\right)=\mathbf{0}, \quad \mathbf{x e}=1 .
$$

Let the row vector $\mathbf{x}$ have the form

$$
\mathbf{x}=\left(\mathbf{x}_{0}, \mathbf{x}_{1}, \ldots, \mathbf{x}_{K}\right),
$$

where $\mathbf{x}_{k}=\widehat{\mathbf{x}}_{k} \otimes \widehat{\mathbf{e}} \otimes \boldsymbol{\theta}, k=\overline{0, K}$. Here, $\widehat{\mathbf{e}}$ is a row vector of size $N-M+1$ with all zero entries except the entry $(\widehat{\mathbf{e}})_{0}$ which is equal to $1, \boldsymbol{\theta}$ is the stationary distribution vector of the process $v_{t}, t \geq 0$, and $\widehat{\mathbf{x}}_{k}, k=\overline{0, K}$ is a row vector of size $M+1$ such as the vector $\left(\widehat{\mathbf{x}}_{0}, \ldots, \widehat{\mathbf{x}}_{K}\right)$ is stochastic.

By direct substitution, we verify that the vector $\mathbf{x}$ from (18) provides the unique solution of system (17) if the vector $\overline{\mathbf{x}}=\left(\overline{\mathbf{x}}_{0}, \overline{\mathbf{x}}_{1}, \ldots, \overline{\mathbf{x}}_{K}\right)$, where $\overline{\mathbf{x}}_{k}=\widehat{\mathbf{x}}_{k} \otimes \boldsymbol{\theta}$ is the unique solution to the following system of linear algebraic equations:

$$
\overline{\mathbf{x}} \overline{\mathbf{Q}}=\overline{\mathbf{x}}, \quad \overline{\mathbf{x}} \mathbf{e}=1 .
$$

Here, $\bar{Q}$ is a block tridiagonal matrix with subdiagonal blocks $\bar{Q}_{k, k-1}$, diagonal blocks $\bar{Q}_{k, k}$, and updiagonal blocks $\bar{Q}_{k, k+1}$ defined by formulas (15).

To calculate the vectors $\overline{\mathbf{x}}_{k}, k=\overline{0, K}$, we use the numerically stable algorithm which consists of the following steps.

Step 1. Calculate the matrices $T_{k}, k=\overline{0, K-1}$, using the backward recursion (14) under the initial condition (13).

Step 2. Calculate the vector $\overline{\mathbf{x}}_{0}$ as the unique solution to the following system:

$$
\begin{aligned}
& \overline{\mathbf{x}}_{0}\left(\bar{Q}_{0,0}+T_{0} \bar{Q}_{1,0}\right)=\mathbf{0}, \\
& \overline{\mathbf{x}}_{0}\left(I+\sum_{k=0}^{K} \prod_{l=0}^{k} T_{l}\right) \mathbf{e}=1 .
\end{aligned}
$$

Step 3. Calculate the vectors $\overline{\mathbf{x}}_{k}=\overline{\mathbf{x}}_{0} \prod_{l=0}^{k} T_{l}, k=\overline{1, K}$.

Substituting the vector $\mathbf{x}$ from (18) into inequality (16) and performing some algebra, we obtain the inequality

$$
\begin{aligned}
\sum_{k=0}^{K}\left(\widehat{\mathbf{x}}_{k} \otimes \boldsymbol{\theta}\right)\left(B \otimes I_{\bar{W}}\right) \mathbf{e} & \\
& >\sum_{k=0}^{K}\left(\widehat{\mathbf{x}}_{k} \otimes \boldsymbol{\theta}\right)\left(I_{M+1} \otimes D_{1}\right) \mathbf{e}
\end{aligned}
$$

or

$$
\sum_{k=0}^{K} \overline{\mathbf{x}}_{k}\left(B \otimes I_{\bar{W}}\right) \mathbf{e}>\sum_{k=0}^{K} \overline{\mathbf{x}}_{k}\left(I_{M+1} \otimes D_{1}\right) \mathbf{e} .
$$

Let the matrix $\mathscr{A}$ be given by formulas (12) and denote the vector $\overline{\mathbf{x}}_{0}$ as $\mathbf{y}$. It is evident that inequality (22) takes form (10) and system (20) is reduced to system (11).

If ergodicity condition (10) of the Markov chain $\xi_{t}$ is fulfilled, then the stationary probabilities of system states $\pi(i, m$, $n, l, \nu), i<N, m=\overline{\max \{0, i-(N-M)\}, \min \{i, M\}}, n=\overline{0, m}$, $l=\overline{0, \min \{i-m, N-M\}}, v=\overline{0, W}$, and $\pi(i, k, n, l, v), i \geq N$, $k=\overline{0, \min \{i-N, K\}}, n=\overline{0, M}, l=\overline{0, N-M}, v=\overline{0, W}$, exist. Let us form the row vectors $\boldsymbol{\pi}_{i}$ of these probabilities enumerated in the lexicographic order of the components $(m, n, l, v)$ if $i<N$ and in the lexicographic order of the components $(k, n, l, v)$ if $i \geq N$.

It is well known that the probability vectors $\pi_{i}, i \geq 0$, satisfy the following system of linear algebraic equations:

$$
\left(\boldsymbol{\pi}_{0}, \boldsymbol{\pi}_{1}, \ldots, \boldsymbol{\pi}_{i}, \ldots\right) \mathrm{Q}=\mathbf{0}, \quad\left(\boldsymbol{\pi}_{0}, \boldsymbol{\pi}_{1}, \ldots, \boldsymbol{\pi}_{i}, \ldots\right) \mathbf{e}=1,
$$

where $Q$ is the infinitesimal generator of the Markov chain $\xi_{t}$, $t \geq 0$.

To solve this system, the following numerically stable algorithm can be used.

Theorem 4. The vectors $\pi_{i}, i \geq 0$, are defined as follows:

$$
\boldsymbol{\pi}_{i}=\pi_{0} \mathscr{F}_{i}, \quad i \geq 1,
$$

where the matrices $\Phi_{i}$ are calculated using the recurrent formulas:

$$
\begin{array}{r}
\mathscr{F}_{0}=I_{\bar{W}}, \quad \mathscr{F}_{i}=-\mathscr{F}_{i-1} Q_{i-1, i}\left(Q_{i, i}+Q_{i, i+1} G_{i}\right)^{-1}, \\
i=\overline{1, N+K-1}, \\
\mathscr{F}_{N+K}=-\mathscr{F}_{N+K-1} Q_{N+K-1, N+K}\left(Q_{1}+Q_{2} G\right)^{-1}, \\
\mathscr{F}_{i}=-\mathscr{F}_{i-1} Q_{0}\left(Q_{1}+Q_{2} G\right)^{-1}, \quad i>N+K,
\end{array}
$$

and the vector $\pi_{0}$ is the unique solution to the system

$$
\boldsymbol{\pi}_{0}\left(Q_{0,0}+Q_{0,1} G_{0}\right)=\mathbf{0}, \quad \boldsymbol{\pi}_{0} \sum_{i=0}^{\infty} \mathscr{F}_{i} \mathbf{e}=1 .
$$

The matrices $G_{i}$ are calculated using the backward recursion

$$
\begin{gathered}
G_{i}=-\left(Q_{i+1, i+1}+Q_{i+1, i+2} G_{i+1}\right)^{-1} Q_{i+1, i}, \\
i=N+K-2, N+K-3, \ldots, 0, \\
G_{N+K-1}=-\left(Q_{1}+Q_{2} G_{N+K}\right)^{-1} Q_{N+K, N+K+1}, \\
G_{N+K}=G,
\end{gathered}
$$

where the matrix $G$ is the minimal nonnegative solution of the matrix equation

$$
Q_{2} G^{2}+Q_{1} G+Q_{0}=O \text {. }
$$

Proof. Proof is based on the results of the paper [8], taking into account the structure of the generator $Q$. 


\section{Performance Measures}

As soon as the vectors $\pi_{i}, i \geq 0$, have been calculated, we are able to find various performance measures of the system under consideration.

The average number of customers in the system is $L=$ $\sum_{i=1}^{\infty} i \pi_{i} \mathbf{e}$. $N\} \boldsymbol{\pi}_{i}$ e.

The average number of busy servers is $N_{\text {busy }}=\sum_{i=1}^{\infty} \min \{i$,

The average number of busy type 1 servers is

$$
N_{1}^{\text {busy }}=\sum_{i=1}^{N-1} \sum_{m=\max \{0, i-(N-M)\}}^{\min \{i, M\}} m \boldsymbol{\pi}(i, m) \mathbf{e}+M \sum_{i=N}^{\infty} \boldsymbol{\pi}_{i} \mathbf{e} .
$$

The average number of busy type 2 servers is

$$
\begin{array}{r}
N_{2}^{\text {busy }}=\sum_{i=1}^{N-1} \sum_{m=\max \{0, i-(N-M)\}}^{\min \{i, M\}}(i-m) \pi(i, m) \mathbf{e} \\
+(N-M) \sum_{i=N}^{\infty} \boldsymbol{\pi}_{i} \mathbf{e} .
\end{array}
$$

The average number of busy servers serving the real customers is

$$
\begin{aligned}
& N_{\text {real }}^{\text {busy }} \\
& =\sum_{i=1}^{N-1} \sum_{m=\max \{0, i-(N-M)\} n=0}^{\min \{i, M\}} \sum_{l=0}^{m} \sum_{i=N}^{m i n\{i-m, N-M\}}(n+l) \pi(i, m, n, l) \mathbf{e} \\
& \quad+\sum_{k=0}^{\infty} \sum_{n=0}^{\min \{i-N, K\}} \sum_{l=0}^{M-M}(n+l) \pi(i, k, n, l) \mathbf{e} .
\end{aligned}
$$

The average number of busy servers serving the virtual customers is

$$
N_{\text {virt }}^{\text {busy }}=N_{\text {busy }}-N_{\text {real }}^{\text {busy }} .
$$
is

The average number of real customers in the finite buffer

$$
N_{\text {buf }}^{\text {real }}=\sum_{i=N}^{\infty} \sum_{k=1}^{\min \{i-N, K\}} k \pi(i, k) \mathbf{e} .
$$

The average number of virtual customers in the infinite buffer is

$$
N_{\text {buf }}^{\mathrm{virt}}=\sum_{i=N}^{\infty} \sum_{k=0}^{\min \{i-N, K\}}(i-k-N) \boldsymbol{\pi}(i, k) \mathbf{e} .
$$

The intensity of flow of real customers, who receive service in the system, is

$$
\lambda_{\text {out }}^{\text {real }}=\mu_{1} N_{\text {busy }}^{\text {real }} .
$$

The intensity of flow of virtual customers, who receive service in the system, is calculated as

$$
\lambda_{\text {out }}^{\text {virt }}=\mu_{2} N_{\text {busy }}^{\text {virt }} \text {. }
$$

The intensity of flow of customers, who receive service in the system, is calculated as

$$
\lambda_{\text {out }}=\lambda_{\text {out }}^{\text {real }}+\lambda_{\text {out }}^{\text {virt }}
$$

The probability $P_{\text {esc }}$ that an arbitrary real customer arrives when all servers are busy, $k, k<K$, real customers present in the buffer, and the customer does not join the buffer and becomes virtual is defined as

$$
P_{\mathrm{esc}}=\lambda^{-1} \sum_{i=N}^{\infty} \sum_{k=0}^{\min \{i-N, K-1\}} p_{k} \boldsymbol{\pi}(i, k)\left(I \otimes D_{1}\right) \mathbf{e} .
$$

The probability $P_{\text {ent }}$ that an arbitrary real customer becomes a virtual customer at the entrance to the system due to the presence of $K$ real customers in the buffer is given by

$$
P_{\text {ent }}=\lambda^{-1} \sum_{i=N+K}^{\infty} \pi(i, K)\left(I \otimes D_{1}\right) \mathbf{e} .
$$

The probability that an arbitrary real customer becomes a virtual customer is calculated as

$$
P_{\text {to-virt }}=1-\frac{\lambda_{\text {out }}^{\text {real }}}{\lambda} \text {. }
$$

The probability that, after arrival, an arbitrary real customer will go to the buffer and leave it due to impatience (becoming a virtual customer) is computed as

$$
P_{\text {imp }}=P_{\text {to-virt }}-P_{\text {ent }}-P_{\text {esc }} \text {. }
$$

\section{Distribution of the Sojourn Time of a Virtual Customer}

To optimize call center operation and to solve the problem of determination of the number of different types operators, it is necessary to know the average sojourn and waiting times of an arbitrary virtual customer. We will derive the distribution of an arbitrary virtual customer's sojourn time in terms of the Laplace-Stieltjes transform (LST).

To derive the expression for the LST $v(s)$ of the distribution of an arbitrary virtual customer's sojourn time in the system, we use the method of collective marks (method of catastrophes; see, e.g., $[9,10]$ ). Let us tag an arbitrary virtual customer and we will keep tracking its stay in the system. Thus, according to the method of catastrophes, $v(s)$ has the meaning of the probability that no catastrophe arrives during the sojourn time of the tagged virtual customer.

Let $v(s, r, k, n, l, v)$ be the probability that a catastrophe will not arrive during the rest of the tagged customer's sojourn time in the system conditioned on the fact that, at the given moment, the position of tagged customer is $r, r \geq 1$, in the buffer for virtual customers, the number of real customers in the buffer is equal to $k, k=\overline{0, K}$, the number of busy type 1 servers that serve real customers is equal to $n, n=\overline{0, M}$, the number of busy type 2 servers that serve real customers is equal to $l, l=\overline{0, N-M}$, and the state of the process $v_{t}, t \geq 0$, is $\nu$. 
Let us enumerate the probabilities $v(s, r, k, n, l, v)$ in the lexicographic order of the components $k, n, l, v$ and form the column vectors

$$
\begin{gathered}
\mathbf{v}(s, r, k, n, l)=(v(s, r, k, n, l, 0), \ldots, v(s, r, k, n, l, W))^{T}, \\
\mathbf{v}(s, r, k, n)=(\mathbf{v}(s, r, k, n, 0), \ldots, \mathbf{v}(s, r, k, n, N-M))^{T}, \\
\mathbf{v}(s, r, k)=(\mathbf{v}(s, r, k, 0), \ldots, \mathbf{v}(s, r, k, M))^{T}, \\
\mathbf{v}(s, r)=(\mathbf{v}(s, r, 0), \ldots, \mathbf{v}(s, r, K))^{T} .
\end{gathered}
$$

Theorem 5. The vectors $\mathbf{v}(s, r), r \geq 1$, are calculated $u$ sing the recurrent formulas

$$
\begin{gathered}
\mathbf{v}(s, 1)=(s I-\Omega)^{-1} \mathbf{a}(s), \\
\mathbf{v}(s, r)=(s I-\Omega)^{-1} \widetilde{\Omega} \mathbf{v}(s, r-1), \quad r>1,
\end{gathered}
$$

where

$$
\begin{aligned}
& \Omega= {\left[N \mu_{2} I_{K+1}+\alpha C_{K+1}\left(I_{K+1}+A_{K+1}\right)\right] } \\
& \otimes I_{(M+1)(N-M+1) \bar{W}}+\left(\mu_{2}-\mu_{1}\right) I_{K+1} \\
& \otimes\left[C_{M+1} \otimes I_{N-M+1}+I_{M+1} \otimes C_{N-M+1}\right] \\
& \otimes I_{\bar{W}}+I_{(K+1)(M+1)(N-M+1)} \otimes D_{0} \\
&+ {\left[\bar{P}+(I-\bar{P}) E_{K+1}^{+}\right] \otimes I_{(M+1)(N-M+1)} \otimes D_{1} } \\
&+ A_{K+1} \otimes\left[\mu_{1} C_{M+1}+\mu_{2} \widetilde{C}_{M+1} E_{M+1}^{+}\right] \otimes I_{(N-M+1) \bar{W}} \\
& \widetilde{\Omega}=I_{(K+1)(M+1)} \otimes\left[\mu_{1} C_{N-M+1} A_{N-M+1}+\mu_{2} \widetilde{C}_{N-M+1}\right] \\
& \otimes I_{\bar{W}}+\bar{I} \otimes\left[\mu_{1} C_{M+1} A_{M+1}+\mu_{2} \widetilde{C}_{M+1}\right] \otimes I_{(N-M+1) \bar{W}}, \\
& \mathbf{a}(s)= {\left[\left(\mu_{1}-\mu_{2}\right) \mathbf{e}_{(K+1)(M+1)} \otimes C_{N-M+1} \mathbf{e} \otimes \mathbf{e}_{\bar{W}}\right.} \\
&+\left(\mu_{1}-\mu_{2}\right) \widetilde{\mathbf{e}} \otimes C_{M+1} \mathbf{e} \otimes \mathbf{e}_{(N-M+1) \bar{W}} \\
&+\left(N \mu_{2} \widetilde{\mathbf{e}}+(N-M) \mu_{2}\left(\mathbf{e}_{K+1}-\widetilde{\mathbf{e}}\right)\right) \\
& \otimes \mathbf{e}_{(M+1)(N-M+1) \bar{W}]} \frac{\mu_{2}}{s+\mu_{2}} .
\end{aligned}
$$

Here,

$C_{l}=\operatorname{diag}\{0,1, \ldots, l-1\}, \widetilde{C}_{l}=\operatorname{diag}\{l-1, l-2, \ldots, 0\}$,

$\bar{P}=\operatorname{diag}\left\{p_{0}, p_{1}, \ldots, p_{K}\right\}$;

$A_{l}$ is a square matrix of sizel with all zero entries except the entries $\left(A_{l}\right)_{i, i-1}, i=\overline{1, l-1}$, which are equal to 1 ;

$\bar{I}$ is a square matrix of size $K+1$ with all zero entries except the entry $(\bar{I})_{0,0}=1$;

$E_{l}^{+}$is a square matrix of sizel with all zero entries except the entries $\left(E_{l}^{+}\right)_{i, i+1}, i=\overline{0, l-1}$, which are equal to 1 ;

$\widetilde{\mathbf{e}}$ is a column vector of size $K+1$ with all zero entries except the entry $(\widetilde{\mathbf{e}})_{0}=1$.
Proof. Based on a probabilistic sense of the LST, we can obtain the following system of linear algebraic equations for calculation of the vectors $\mathbf{v}(s, r, k, n, l)$ :

$$
\begin{aligned}
& \mathbf{v}(s, r, k, n, l) \\
& =\left[\left(s+k \alpha+(n+l) \mu_{1}\right.\right. \\
& \left.\left.+(N-(n+l)) \mu_{2}\right) I_{\bar{W}}-D_{0}\right]^{-1} \\
& \times\left(\delta _ { r , 1 } \left[\delta_{k, 0}\left((n+l) \mu_{1}+(N-(n+l)) \mu_{2}\right)\right.\right. \\
& \left.+\left(1-\delta_{k, 0}\right)\left(l \mu_{1}+(N-M-l) \mu_{2}\right)\right] \\
& \times \frac{\mu_{2}}{s+\mu_{2}} \mathbf{e}_{\bar{W}}+\left(1-\delta_{r, 1}\right) \\
& \times\left[\delta _ { k , 0 } \left(n \mu_{1} \mathbf{v}(s, r-1,0, n-1, l)\right.\right. \\
& \left.+(M-n) \mu_{2} \mathbf{v}(s, r-1,0, n, l)\right) \\
& +l \mu_{1} \mathbf{v}(s, r-1, k, n, l-1) \\
& \left.+(N-M-l) \mu_{2} \mathbf{v}(s, r-1, k, n, l)\right] \\
& +\left(1-\delta_{k, 0}\right) \times\left(\left(k \alpha+n \mu_{1}\right) \mathbf{v}(s, r, k-1, n, l)\right. \\
& \left.+(M-n) \mu_{2} \mathbf{v}(s, r, k-1, n+1, l)\right) \\
& +\left(1-p_{k}\right) D_{1} \mathbf{v}(s, r, k+1, n, l) \\
& \left.+p_{k} D_{1} \mathbf{v}(s, r, k, n, l)\right) \text {, } \\
& l=\overline{0, N-M}, \quad n=\overline{0, M}, \quad k=\overline{0, K}, \quad r \geq 1,
\end{aligned}
$$

where $\delta_{i, j}$ indicates the Kronecker delta.

Let us explain formula (45) in brief. The diagonal entries of the matrix in the first square brackets in (45) are equal to the total intensity of the events which can happen after an arbitrary epoch: catastrophe arrival, transition of the directing process of the MAP, and abandon of real customer from the buffer, the service completion of real or virtual customers. The nondiagonal entries of this matrix are equal to the intensities of the events which do not impact the components $s, r, k, n$, and $l$ (transition of the MAP underlying process without generation of a customer). The first term in the round brackets in (45) corresponds to the case when the tagged customer has the first position in the buffer, one service process is completed and a virtual customer is chosen for service. In this case, the tagged customer is chosen for service, and the number $\mu_{2} /\left(s+\mu_{2}\right)$ defines the probability that a catastrophe will not arrive during the service time of the tagged customer. The second term corresponds to the case when the position of the tagged customer in the buffer is greater than 1, one service process is completed, and a virtual customer is chosen for service. In this case, the position of the tagged customer in the buffer decreases by 1 . The third term corresponds to the cases when the service process is completed and a real customer is chosen for service or a real customer leaves the buffer due to impatience. In this case, the number of real customers in the buffer decreases by 1 . The 
fourth term corresponds to the case when a new customer arrives and is admitted to the system. In this case, the number of real customers in the buffer increases by 1 . The fifth term corresponds to the case when a real customer arrives to the system and does not attend the buffer. In this case, the values of the components $s, r, k, n$, and $l$ do not change.

Using the notation of the matrices given in Theorem 5, let us rewrite system (45) into the matrix form as follows:

$$
\begin{aligned}
& {\left[-\left(s+k \alpha-n\left(\mu_{2}-\mu_{1}\right)+N \mu_{2}\right) I_{(N-M+1) \bar{W}}\right.} \\
& +\left(\mu_{2}-\mu_{1}\right) C_{N-M+1} \otimes I_{\bar{W}}+I_{N-M+1} \\
& \left.\otimes\left(D_{0}+p_{k} D_{1}\right)\right] \mathbf{v}(s, r, k, n) \\
& +\delta_{r, 1}\left[\left(\mu_{1}-\mu_{2}\right) C_{N-M+1} \mathbf{e} \otimes \mathbf{e}_{\bar{W}}\right. \\
& +\left(\delta_{k, 0}\left(n\left(\mu_{1}-\mu_{2}\right)+N \mu_{2}\right)\right. \\
& \left.\left.+\left(1-\delta_{k, 0}\right)(N-M) \mu_{2}\right) \mathbf{e}_{(N-M+1) \bar{W}}\right] \\
& \times \frac{\mu_{2}}{s+\mu_{2}}+\left(1-\delta_{r, 1}\right) \\
& \times\left[\left(\mu_{1} C_{N-M+1} A_{N-M+1}+\mu_{2} \widetilde{C}_{N-M+1}\right)\right. \\
& \otimes I_{\bar{W}} \mathbf{v}(s, r-1, k, n) \\
& +\delta_{k, 0}\left(n \mu_{1} \mathbf{v}(s, r-1,0, n-1)\right. \\
& \left.\left.+(M-n) \mu_{2} \mathbf{v}(s, r-1,0, n)\right)\right] \\
& +\left(1-\delta_{k, 0}\right)\left[\left(k \alpha+n \mu_{1}\right) \mathbf{v}(s, r, k-1, n)\right. \\
& \left.+(M-n) \mu_{2} \mathbf{v}(s, r, k-1, n+1)\right] \\
& +\left(1-p_{k}\right) I_{N-M+1} \otimes D_{1} \mathbf{v}(s, r, k+1, n)=\mathbf{0}^{T} \text {. }
\end{aligned}
$$

System (46) can be further rewritten into

$$
\begin{gathered}
\left\{-\left(s+k \alpha+N \mu_{2}\right) I_{(M+1)(N-M+1) \bar{W}}\right. \\
+\left(\mu_{2}-\mu_{1}\right)\left[C_{M+1} \otimes I_{N-M+1}+I_{M+1} \otimes C_{N-M+1}\right] \\
\left.\otimes I_{\bar{W}}+I_{(M+1)(N-M+1)} \otimes\left(D_{0}+p_{k} D_{1}\right)\right\} \mathbf{v}(s, r, k) \\
+\delta_{r, 1}\left\{\left(\mu_{1}-\mu_{2}\right) \mathbf{e}_{M+1} \otimes C_{N-M+1} \mathbf{e}\right. \\
\otimes \mathbf{e}_{\bar{W}}+\delta_{k, 0}\left(\left(\mu_{1}-\mu_{2}\right) C_{M+1} \mathbf{e} \otimes \mathbf{e}_{(N-M+1) \bar{W}}\right. \\
\left.+N \mu_{2} \mathbf{e}_{(M+1)(N-M+1) \bar{W}}\right) \\
\left.+\left(1-\delta_{k, 0}\right) \mu_{2}(N-M) \mathbf{e}_{(M+1)(N-M+1) \bar{W}}\right\} \frac{\mu_{2}}{s+\mu_{2}} \\
+\left(1-\delta_{r, 1}\right)\left\{I_{M+1} \otimes\left[\mu_{1} C_{N-M+1} A_{N-M+1}+\mu_{2} \widetilde{C}_{N-M+1}\right]\right. \\
\otimes I_{\bar{W}} \mathbf{v}(s, r-1, k) \\
+\delta_{k, 0}\left(\mu_{1} C_{M+1} A_{M+1}+\mu_{2} \widetilde{C}_{M+1}\right)
\end{gathered}
$$

$$
\begin{gathered}
\left.\otimes I_{(N-M+1) \bar{W}} \mathbf{v}(s, r-1,0)\right\} \\
+\left(1-\delta_{k, 0}\right)\left(k \alpha I_{M+1}+\mu_{1} C_{M+1}+\mu_{2} \widetilde{C}_{M+1} E_{M+1}^{+}\right) \\
\otimes I_{(N+M-1) \bar{W}} \mathbf{v}(s, r, k-1)+\left(1-p_{k}\right) I_{(M+1)(N-M+1)} \\
\otimes D_{1} \mathbf{v}(s, r, k+1)=\mathbf{0}^{T}
\end{gathered}
$$

Taking into account notations (44), performing algebra, we obtain the following system:

$$
\begin{array}{r}
(-s I+\Omega) \mathbf{v}(s, r)+\left(1-\delta_{r, 1}\right) \widetilde{\Omega} \mathbf{v}(s, r-1)+\delta_{r, 1} \mathbf{a}(s)=\mathbf{0}^{T}, \\
r \geq 1 .
\end{array}
$$

Based on system (48), it is easy to verify that the vectors $\mathbf{v}(s, r), r \geq 1$, can be calculated using formulas (43).

Based on a probabilistic sense of the LST and the law of total probability, one can prove the following assertion.

Theorem 6. The LST $v(s)$ of the distribution of an arbitrary virtual customer's sojourn time in the system is computed as

$$
\begin{aligned}
& v(s)=\frac{1}{\lambda_{\text {out }}^{\text {virt }}}\left\{\sum_{i=N}^{\infty} \sum_{k=0}^{\min \{i-N, K\}} \sum_{n=0}^{M} \sum_{l=0}^{N-M} p_{k} \pi(i, k, n, l) D_{1}\right. \\
& \times \mathbf{v}(s, i-N-k \\
& +1, k, n, l) \\
& +\sum_{i=N}^{\infty} \sum_{k=1}^{\min \{i-N, K\}} \sum_{n=0}^{M} \sum_{l=0}^{N-M} k \alpha \pi(i, k, n, l) \\
& \times \mathbf{v}(s, i-N-k+1, \\
& k-1, n, l)\} \text {. }
\end{aligned}
$$

Corollary 7. The average sojourn time $V^{\text {soj }}$ of an arbitrary virtual customer is computed by

$$
\begin{array}{r}
V^{\text {soj }}=-\frac{1}{\lambda_{\text {out }}^{\text {virt }}}\left\{\sum_{i=N}^{\infty} \sum_{k=0}^{\min \{i-N, K\}} \sum_{n=0}^{M} \sum_{l=0}^{N-M} p_{k} \pi(i, k, n, l) D_{1}\right. \\
\times \mathbf{v}^{\prime}(s, i-N-k \\
+1, k, n, l)\left.\right|_{s=0} \\
+\sum_{i=N}^{\infty} \sum_{k=1}^{\infty} \sum_{n=0}^{\min \{i-N, K\}} \sum_{l=0}^{M-M} k \alpha \pi(i, k, n, l) \\
\times \mathbf{v}^{\prime}(s, i-N-k \\
\left.+1, k-1, n, l)\left.\right|_{s=0}\right\} .
\end{array}
$$


Here, the column vectors $\left.\mathbf{v}^{\prime}(s, r, k, n, l)\right|_{s=0}$ are calculated as the blocks of the vector $\left.\mathbf{v}^{\prime}(s, r)\right|_{s=0}, r \geq 1$, which can be computed as follows:

$$
\begin{gathered}
\left.\mathbf{v}^{\prime}(s, 1)\right|_{s=0}=\Omega^{-1}\left[\mathbf{e}-\left.\mathbf{a}^{\prime}(s)\right|_{s=0}\right], \\
\left.\mathbf{v}^{\prime}(s, r)\right|_{s=0}=\Omega^{-1}\left[\mathbf{e}-\left.\widetilde{\Omega} \mathbf{v}^{\prime}(s, r-1)\right|_{s=0}\right], \quad r>1 .
\end{gathered}
$$

Proof. Formula (50) is based on the definition $V^{\text {soj }}=$ $-\left.v^{\prime}(s)\right|_{s=0}$, taking into account that $\left.\mathbf{v}(s, r)\right|_{s=0}=\mathbf{e}, r \geq 1$.

Corollary 8. The average waiting time $V^{\text {wait }}$ of an arbitrary virtual customer is defined as

$$
V^{\text {wait }}=V^{s o j}-\frac{1}{\mu_{2}} .
$$

\section{Numerical Examples}

Experiment 1. In this experiment we investigate the impact of the correlation and variation in the arrival flow on the system performance measures.

For this purpose, let us introduce four MAPs, defined by the matrices $D_{0}$ and $D_{1}$, with the same average arrival rate $\lambda=9$ but different coefficients of correlation and variation.

The first process, coded as $M^{0}$, is a stationary Poisson process. It is defined by the matrices $D_{0}=(-9)$ and $D_{1}=$ (9) and has the coefficient of correlation $c_{\text {cor }}=0$ and the coefficient of variation $c_{\mathrm{var}}=1$.

The second process, coded as $\operatorname{IPP}^{0}$ (interrupted Poisson process), is defined by the matrices

$$
\begin{gathered}
D_{0}=\left(\begin{array}{cc}
-28.64540 & 0.27584 \\
0.97606 & -0.97606
\end{array}\right), \\
D_{1}=\left(\begin{array}{cc}
26.54474 & 1.82482 \\
0 & 0
\end{array}\right) .
\end{gathered}
$$

It has the coefficient of correlation $c_{\text {cor }}=0$ and the coefficient of variation $c_{\mathrm{var}}=12.4$.

The third process, coded as $\mathrm{MAP}^{0.2}$, defined by the matrices

$$
\begin{gathered}
D_{0}=\left(\begin{array}{cc}
-12.16476 & 0 \\
0 & -0.39483
\end{array}\right), \\
D_{1}=\left(\begin{array}{cc}
12.08388 & 0.08088 \\
0.21991 & 0.17492
\end{array}\right)
\end{gathered}
$$

and has $c_{\mathrm{cor}}=0.2$ and $c_{\mathrm{var}}=12.34$.

The fourth process, coded as $\mathrm{MAP}^{0.4}$, is defined by the matrices

$$
\begin{gathered}
D_{0}=\left(\begin{array}{cc}
-30.57918 & 0 \\
0.00913 & -0.99199
\end{array}\right), \\
D_{1}=\left(\begin{array}{cc}
30.26068 & 0.31850 \\
0.10919 & 0.87367
\end{array}\right) .
\end{gathered}
$$

It has $c_{\text {cor }}=0.4$ and $c_{\text {var }}=12.39$.
We assume that the number of servers $N$ is equal to 15 , the service intensities of real and virtual customers $\mu_{1}=0.7$ and $\mu_{2}=0.8$, respectively, the buffer capacity $K=15$, the intensity of impatience $\alpha=0.3$, and the probabilities $p_{k}=$ $0.02(k+1), k=\overline{0,14}$.

Let us vary the number of type 1 servers $M$ in the interval $[0,15]$.

Figure 2 illustrates the dependence of the average number of customers in the system $L$, the average waiting time of a virtual customer $V^{\text {wait }}$, the average number of real customers $N_{\text {buf }}^{\text {real }}$, and the average number of virtual customers $N_{\text {buf }}^{\text {virt }}$ in the buffer on the number of type 1 servers $M$ for the four MAP arrival processes presented above.

The dependence of the intensity of flow of served real customers $\lambda_{\text {out }}^{\text {real }}$, the intensity of flow of served virtual customers $\lambda_{\text {out }}^{\text {virt }}$, the probability $P_{\text {ent }}$ that an arbitrary real customer becomes a virtual customer at the entrance to the system due to the presence of $K$ real customers in the buffer and the probability $P_{\text {imp }}$ that an arbitrary real customer after arrival will go to the buffer and leave it due to impatience on the number of type 1 servers $M$ for different MAP arrival processes is presented in Figure 3.

From Figures 2 and 3, one can conclude that the coefficients of correlation and variation in the arrival process have a profound impact on the system performance measures. As the coefficients of correlation and variation increase, the intensities $\lambda_{\text {out }}^{\text {virt }}$ and $\lambda_{\text {out }}^{\text {real }}$ decrease essentially, while the average number of customers in the system $L$, the average waiting time of an arbitrary virtual customer $V_{\text {wait }}$, and the loss probability $P_{\text {ent }}$ increase. Thus, increasing the coefficients of correlation and variation has a negative effect on system operation.

Additionally, one can conclude, that with increasing the number of type 1 servers $M$ the intensity $\lambda_{\text {out }}^{\text {real }}$ increases, while intensity $\lambda_{\text {out }}^{\text {virt }}$ decreases. However, at the same time, the average waiting time of an arbitrary virtual customer $V_{\text {wait }}$ increases essentially with increasing $M$, that has a negative impact on the quality of service of virtual customers.

The results of this experiment also show that Little's formula holds true for the system under study; that is,

$$
V^{\text {wait }}=\frac{N_{\text {virt }}^{\text {buffer }}}{\lambda P_{\text {to-virt }}}=\frac{N_{\text {virt }}^{\text {buffer }}}{\lambda_{\text {out }}^{\text {virt }}} \text {. }
$$

Additionally, the numerical results show that another equality holds true: $N_{\text {buf }}^{\text {real }} / \lambda=P_{\text {imp }} / \alpha$. To prove this equality, let us rewrite it in the form $\alpha N_{\text {buf }}^{\text {real }}=\lambda P_{\text {imp }}$. The left and right sides of this equality define the intensity of real customers' leaving the buffer due to impatience, so the equality is correct.

Experiment 2. The issue of the optimal choice of the number of each type operator is very important for the effective organization of the call center performance. In this experiment, we numerically solve the problem of optimal choice of the number of type 1 servers $M$. The aim of optimization is the maximization of a cost criterion (one of the possible criteria):

$$
\Lambda(M)=\lambda_{\text {out }}^{\text {real }}+\left(1-q_{1}\right) q_{2} \lambda_{\text {out }}^{\text {virt }}
$$




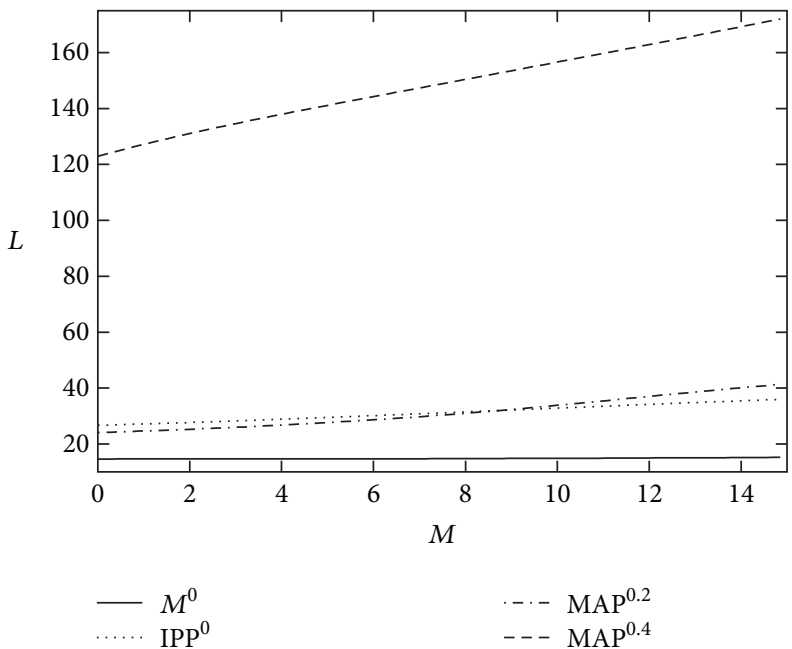

(a)

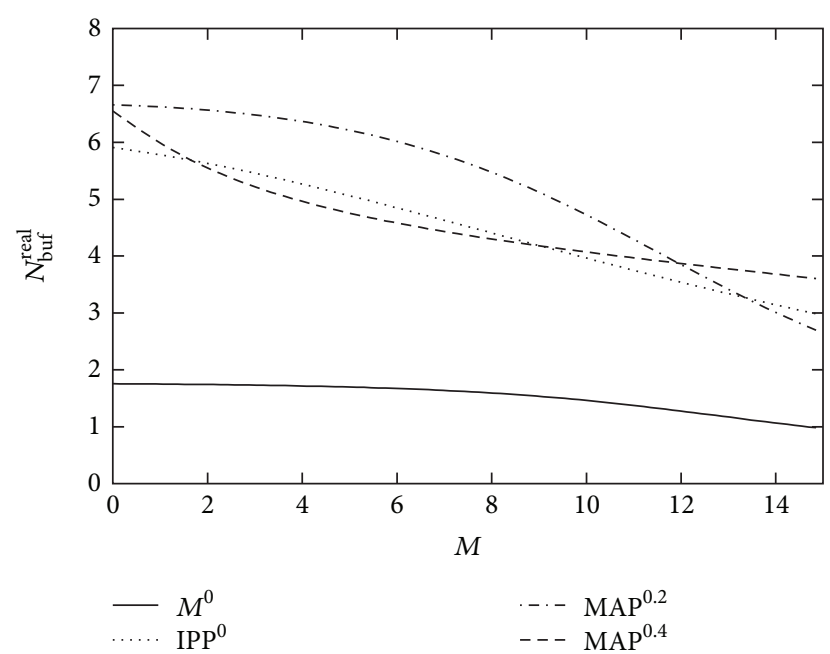

(c)

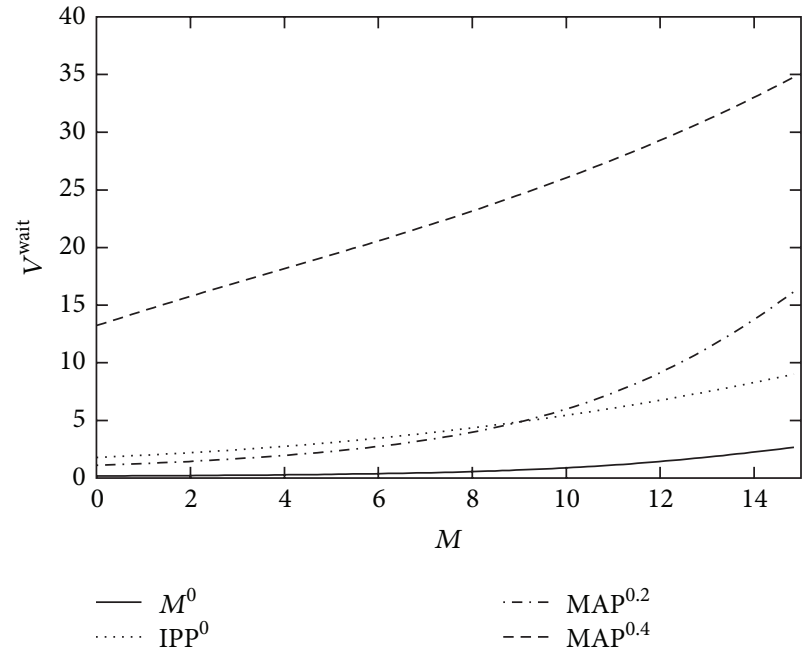

(b)

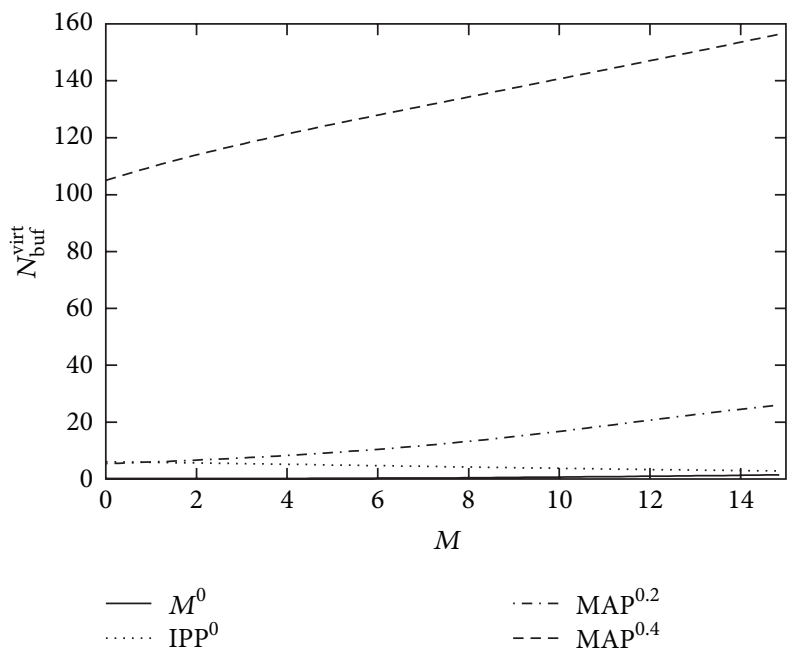

(d)

Figure 2: Average number of customers in the system, the average waiting time of a virtual customer, and the average number of real and virtual customers in the buffer as functions of the number of type 1 servers for different MAPs.

Here, $q_{1}$ is the probability that an operator will not succeed to connect to a customer (customer's phone is busy or does not answer) and $q_{2}$ is the probability that a virtual customer will be interested in the services proposed by an operator. It is evident that the probability $q_{2}$ depends on the waiting time. Thus, $\Lambda(M)$ determines the effective intensity of the flow of customers, who successfully receive service in the system.

Let us fix $q_{1}=0.1$, and

$$
q_{2}= \begin{cases}0, & V^{\text {wait }} \geq 40, \\ 1-\frac{V^{\text {wait }}}{40}, & V^{\text {wait }}<40 .\end{cases}
$$

Our goal is to find the optimal value $M^{*}$ that provides the maximum value of the cost criterion $\Lambda(M)$. The value of the cost criterion $\Lambda(M)$ for different values of the number of type 1 servers $M$ and different arrival processes is presented in
Table 1. The optimal values of $\Lambda(M)$ for each arrival process are printed in bold type. Additionally, Table 1 contains the values of the cost criterion $\Lambda(M)$ for the call center that does not provide a call-back option. In this case, $\Lambda(M)=\lambda_{\text {out }}$ and can be found based on the results of the paper [11].

As it is seen from Table 1, for the stationary Poisson arrival process, the maximum value of $\Lambda(M)$ is equal to 8.91163 for $M=15$; that is, when all operators are of type 1 and there are no type 2 operators. For the interrupted Poisson process, the maximum value of $\Lambda(M)$ is equal to 8.32646 , when the system has seven type 1 operators and eight type 2 operators. For MAP ${ }^{0.2}$ arrival process, the maximum value of $\Lambda(M)$ is equal to 8.39144 , when the system has three type 1 operators and twelve type 2 operators and for $\mathrm{MAP}^{0.4}$ arrival process the maximum value of $\Lambda(M)$ is equal to 5.84454 when the system has only type 2 operators. 


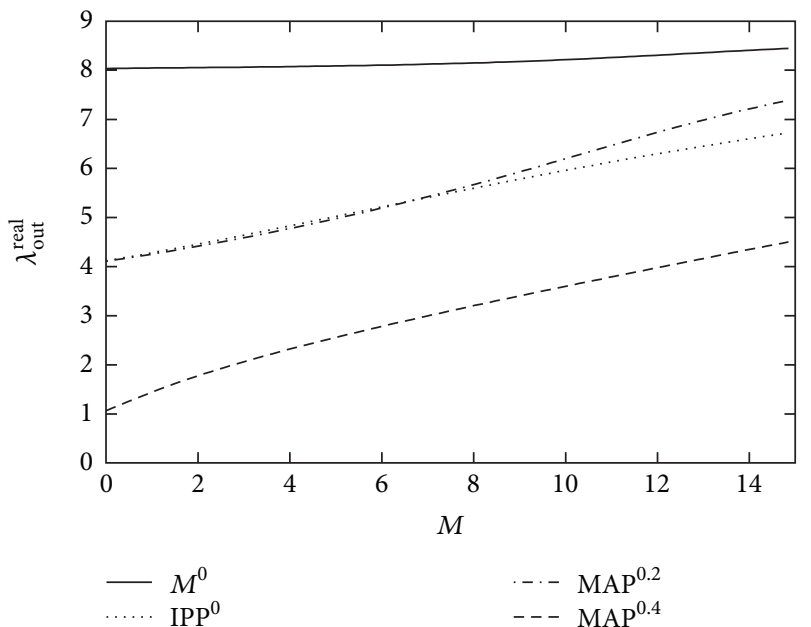

(a)

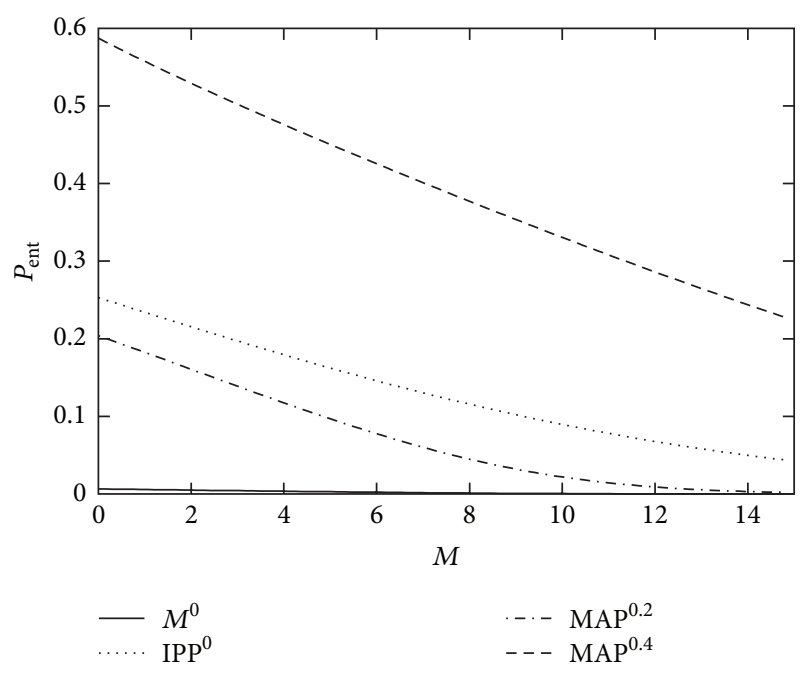

(c)

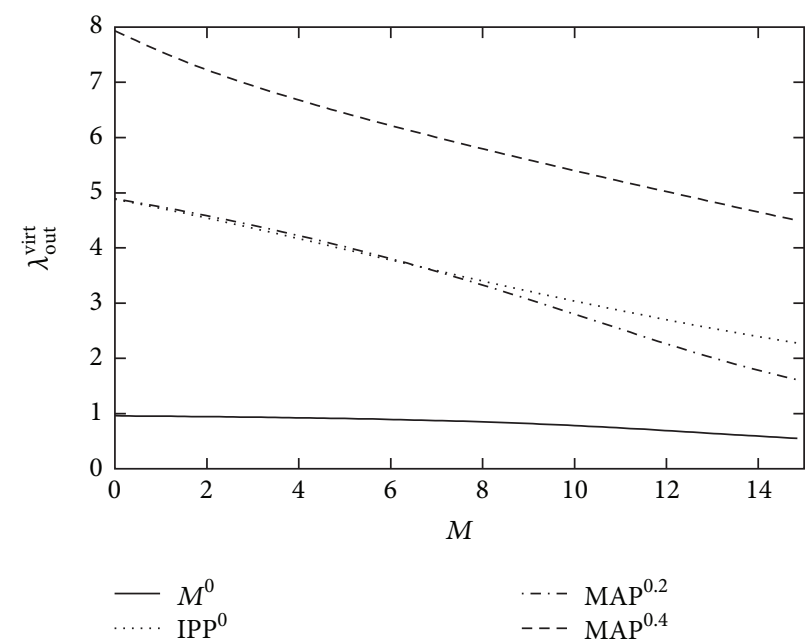

(b)

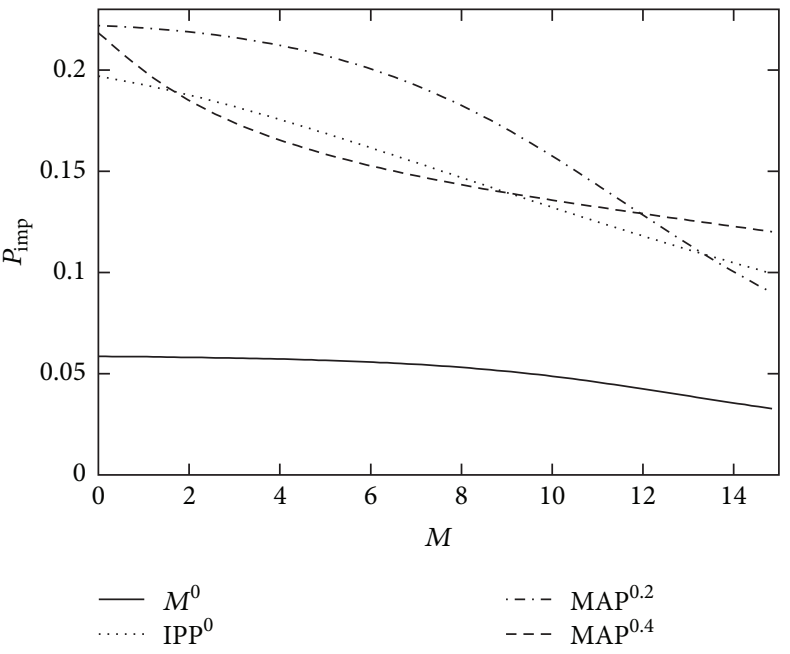

(d)

FIGURE 3: Intensities of output flow of real and virtual customers, who received service in the system, and the loss probabilities $P_{\text {ent }}$ and $P_{\text {imp }}$ as functions of the number of type 1 servers $M$ for different MAPs.

From Table 1, one can conclude that providing of a callback for lost customers makes sense and delivers benefits to a call center. For any choice of the number of type 1 operators, the values of $\Lambda(M)$ are essentially higher for the system with call-backs than without call-backs. Another important conclusion is that the coefficients of correlation and variation in the arrival process strongly affect the values of cost criterion $\Lambda(M)$ and the values of the optimal number of type 1 operators. So, for the correct prediction of system performance measures it is necessary to take into account the coefficients of correlation and variation in the arrival process.

\section{Conclusion}

In this paper, a novel multiserver queueing system with impatient heterogeneous customers and two types of servers, which can be suited for modeling a real-life call center with call-backs for lost customers, is investigated. The ergodicity condition is derived. An efficient algorithm for calculating the stationary probabilities of the system states is presented. The main performance measures and the Laplace-Stieltjes transform of the distribution of the sojourn time of an arbitrary virtual customer are derived. The numerical results confirm the importance of the analysis of queueing models with the Markovian arrival process because correlation and variation in the arrival process essentially impact system operation. The problem of optimal choice of the number of each type servers is solved numerically.

\section{Acknowledgment}

This research was supported by the Basic Science Research Program through the National Research Foundation of Korea 
TABLE 1: Value of the flow intensity of customers, who receive service in the system, for different values of the number of type 1 servers $M$ and different arrival processes.

\begin{tabular}{|c|c|c|c|c|}
\hline$\Lambda(M)$ & $M^{0}$ & $\mathrm{IPP}^{0}$ & $\mathrm{MAP}^{0.2}$ & $\mathrm{MAP}^{0.4}$ \\
\hline Without call-back & 8.59707 & 7.22160 & 7.76157 & 4.89795 \\
\hline$M=0$ & 8.89987 & 8.31396 & 8.38843 & 5.84454 \\
\hline$M=1$ & 8.90027 & 8.31731 & 8.39022 & 5.77657 \\
\hline$M=2$ & 8.90068 & 8.32023 & 8.39130 & 5.71393 \\
\hline$M=3$ & 8.90112 & 8.32264 & 8.39144 & 5.65655 \\
\hline$M=4$ & 8.90158 & 8.32449 & 8.39039 & 5.60255 \\
\hline$M=5$ & 8.90208 & 8.32575 & 8.38787 & 5.55049 \\
\hline$M=6$ & 8.90263 & 8.32640 & 8.38354 & 5.49944 \\
\hline$M=7$ & 8.90325 & 8.32646 & 8.37706 & 5.44876 \\
\hline$M=8$ & 8.90396 & 8.32595 & 8.36812 & 5.39803 \\
\hline$M=9$ & 8.9048 & 8.32490 & 8.35651 & 5.34692 \\
\hline$M=10$ & 8.90577 & 8.32335 & 8.3422 & 5.29522 \\
\hline$M=11$ & 8.90688 & 8.32135 & 8.3255 & 5.24273 \\
\hline$M=12$ & 8.90809 & 8.31894 & 8.30702 & 5.18933 \\
\hline$M=13$ & 8.90932 & 8.31617 & 8.28766 & 5.13492 \\
\hline$M=14$ & 8.91052 & 8.31309 & 8.26835 & 5.07942 \\
\hline$M=15$ & 8.91163 & 8.30977 & 8.24991 & 5.02276 \\
\hline
\end{tabular}

(NRF) funded by the Ministry of Education, Science and Technology (Grant no. 2011-0015214).

\section{References}

[1] O. Z. Aksin, M. Armony, and V. Mehrotra, "The modern call center: a multi-disciplinary perspective on operations management research," Production and Operations Management, vol. 16, no. 6, pp. 665-688, 2007.

[2] J. W. Kim and S. C. Park, "Outsourcing strategy in two-stage call centers," Computers and Operations Research, vol. 37, no. 4, pp. 790-805, 2010.

[3] C. Kim, S. Dudin, O. Taramin, and J. Baek, "Queueing system $M A P|P H| N \mid N+R$ with impatient heterogeneous customers as a model of call center," Applied Mathematical Modelling, vol. 37, no. 3, pp. 958-976, 2013.

[4] M. Armony and C. Maglaras, "On customer contact centers with a call-back option: customer decisions, routing rules, and system design," Operations Research, vol. 52, no. 2, pp. 271-292, 2004.

[5] M. Armony and C. Maglaras, "Contact centers with a call-back option and real-time delay information," Operations Research, vol. 52, no. 4, pp. 527-545, 2004.

[6] C. Kim, O. Dudin, A. Dudin, and S. Dudin, "Queueing system $M A P|M| N$ as a model of call center with call-back option," Lecture Notes in Computer Science, vol. 7314, pp. 1-15, 2012.

[7] M. Neuts, Matrix-Geometric Solutions in Stochastic Models. An Algorithmic Approach, vol. 2, Johns Hopkins University Press, Baltimore, Md, USA, 1981.

[8] V. Klimenok and A. Dudin, "Multi-dimensional asymptotically quasi-Toeplitz Markov chains and their application in queueing theory," Queueing Systems, vol. 54, no. 4, pp. 245-259, 2006.

[9] H. Kesten and J. T. Runnenburg, Priority in Waiting Line Problems, Mathematisch Centrum, Amsterdam, The Netherlands, 1956.
[10] D. van Dantzig, "Chaînes de Markof dans les ensembles abstraits et applications aux processus avec régions absorbantes et au problème des boucles," Annales de l'Institut Henri Poincaré, vol. 14, pp. 145-199, 1955.

[11] S. A. Dudin and O. S. Dudina, "A call center operation model as a $M A P|P H| N \mid R-N$ system with impatient customers," Problems of Information Transmission, vol. 47, pp. 364-377, 2011. 


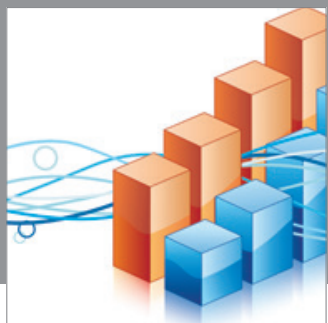

Advances in

Operations Research

mansans

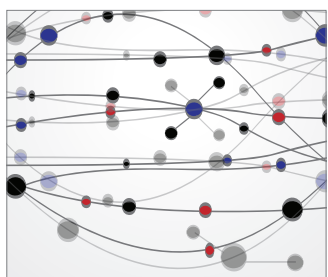

The Scientific World Journal
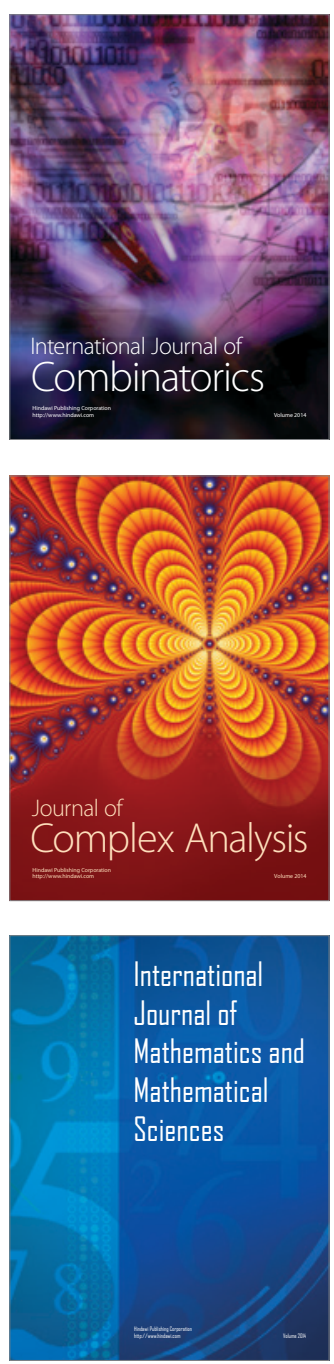
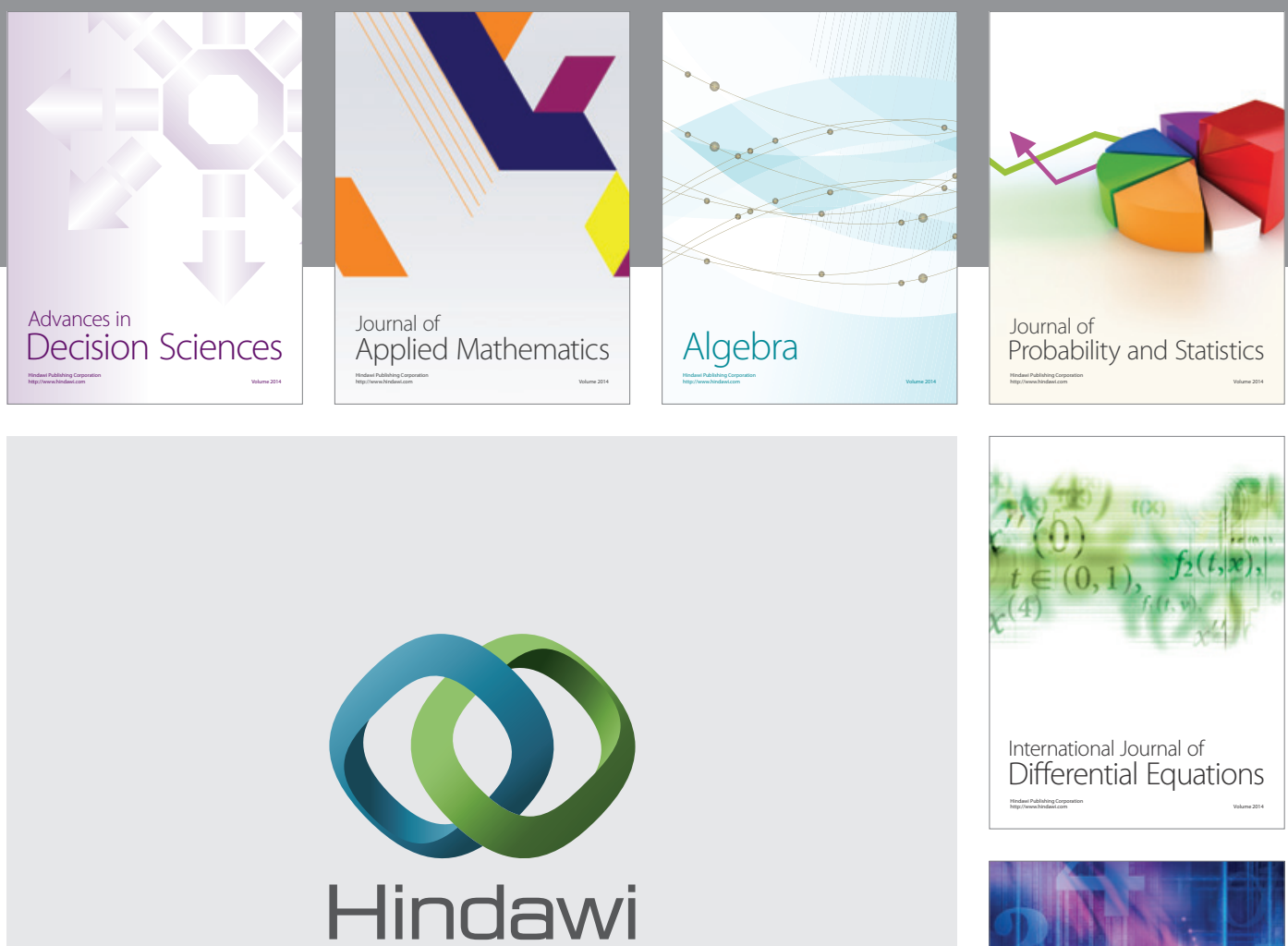

Submit your manuscripts at http://www.hindawi.com
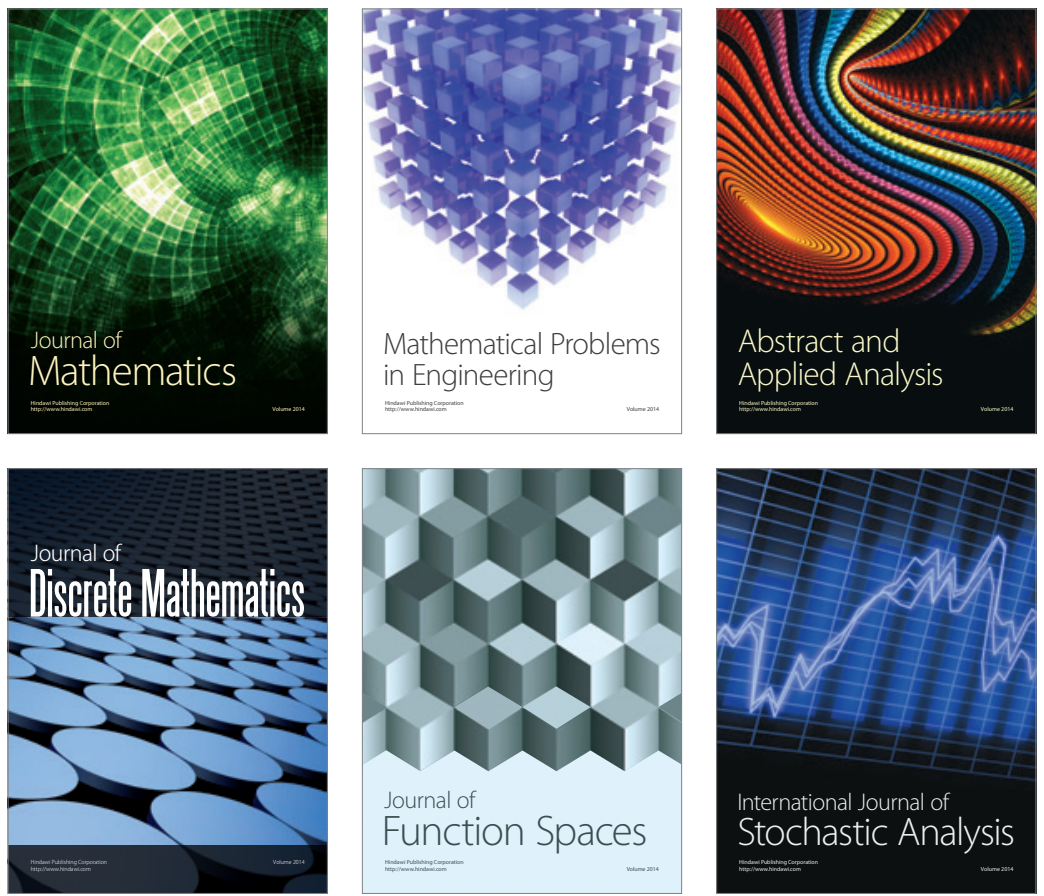

Journal of

Function Spaces

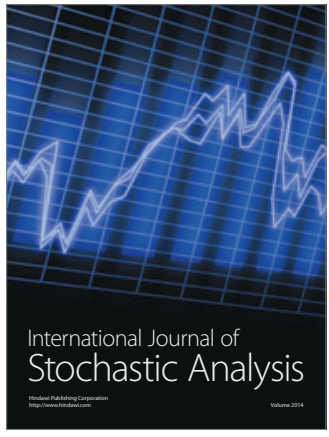

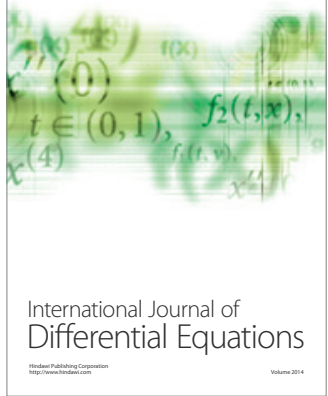
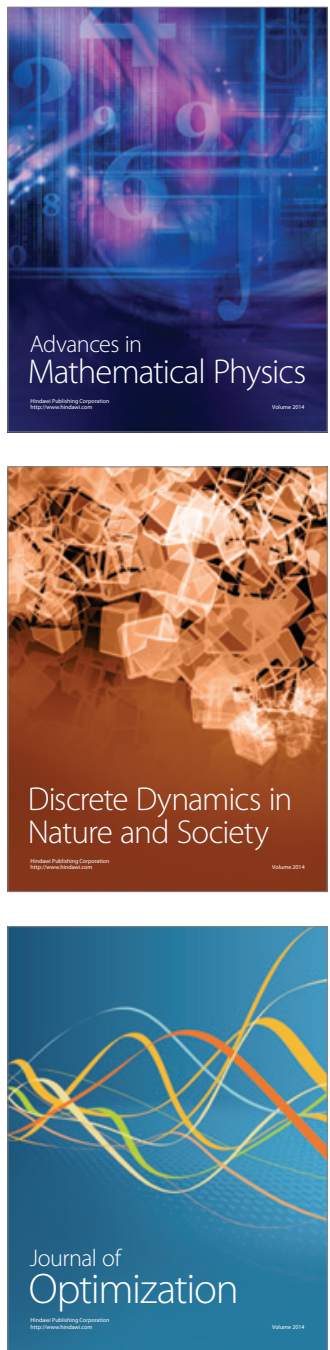